\title{
EFEITOS DA RADIAÇÃO GAMA DO COBALTO-60 EM OVOS DE Tribolium castaneum (HERBST., 1797) (COLEOPTERA: TENEBRIONIDAE)
}

\author{
L. S. FONTES; V. ARTHUR \\ Centro de Energia Nuclear na Agricultura/USP - C.P. 96 - CEP: 13416-970 - Piracicaba, SP.
}

\begin{abstract}
RFSUMO: Este trabalho teve por objetivo determinar as doses esterilizantes e letal para ovos de $T$. castaneum (Herbst., 1797) (Coleoptera: Tenebrionidae), através de doses crescentes de radiação gama. Utilizou-se uma fonte de Cobalto-60, tipo Gammabeam-650, com taxa de dose de 1,28 kGy/hora. 0 experimento foi conduzido sob condições controladas com temperatura de $25 \pm 2^{\circ} \mathrm{C}$ e umidade relativa de $70 \pm 5 \%$. As doses letal e esterilizante para os ovos foram respectivamente 30 e $20 \mathrm{~Gy}$.
\end{abstract}

Descritores: radiação gama, ovos, Tribolium castaneum

\section{GAMMA RADIATION EFFECTS ON EGGS OF Tribolium castaneum (HERBST., 1797) (COLEOPTERA: TENEBRIONIDAE)}

\begin{abstract}
SUMMARY: The objective of this research was to verify the effects of gamma radiation of a Cobalt-60 source on eggs of the red flour beetle Tribolium castaneum (Herbst., 1797). The used dose rate was $1.28 \mathrm{kGy}$ per hour, and the irradiated insects were kept under controlled environment condition: $25 \pm 2{ }^{\circ} \mathrm{C}$ and $70 \pm 5 \%$ relative humidity. For eggs the sterilizing and lethal dose were respectively, 20 and $30 \mathrm{~Gy}$.

Key Words: gamma radiation, eggs, Tribolium castaneum
\end{abstract}

\section{INTRODUÇÃO}

Tendo em vista os elevados danos causados aos grãos e produtos armazenados pelos insetos, torna-se necessário por em prática meios de controle, a fim de se evitar os prejuízos. Um dos métodos mais utilizados é a aplicação de produtos químicos, que apresenta vários inconvenientes, entre eles a possibilidade de causar intoxicação ao consumidor por deixar resíduos nos alimentos tratados. Por ser um método livre de resíduos para o controle de pragas, o tratamento com radiação é um substituto viável à fumigação para satisfazer os regulamentos quarentenários de vários países.

RUNNER (1969), pela primeira vez obteve êxito na esterilização do caruncho do fumo Lasioderma serricone ( $\mathrm{F}$.) utilizando raios-X.

No Brasil, os primeiros estudos foram realizados por GALLO (1960), irradiando pupas de Ceratitis capitata (Wied.) e Diatraea saccharalis (F.), com irradiação gama proveniente do berílio, com o objetivo de obter insetos estéreis. Ainda no Brasil, somente WIENDL (1969), trabalhando com Zabrotes subfasciatus (Boh.), iniciou o problema de desinfestação por radiações ionizantes.
Segundo GALLO et al. (1988), entre os insetos que causam grandes perdas qualitatitvas e quantitativas, destaca-se o Tribolium castaneum (Coleoptera: Tenebrionidae), que ataca todos os tipos de cereais moídos, como farelos, rações, farinhas, fubá e grãos quebrados, defeituosos ou já atacados por outras pragas. Praticamente todo o tipo de farinha pode ser infestada por Tribolium castaneum e a farinha de trigo integral parece ser, especialmente a mais propensa ao ataque do inseto.

Devido a importância dessa praga para produtos armazenados, o presente trabalho teve como objetivo determinar as doses esterilizantes e letais de radiação gama do Cobalto-60 para a fase de ovo de $T$. castaneum em farinha de trigo visando o seu controle (desinfestação).

\section{REVISÃO BIBLIOGRÁFICA}

CROOCK (1962) verificou a susceptibilidade de todos os estádios de desenvolvimento de $T$. castaneum (Herbst.) à radiação gama, observou que as doses de $11.100 \mathrm{rad}, 10.800 \mathrm{rad}$ e $21.900 \mathrm{rad}$, causaram a mortalidade de ovos, larvas, pupas e adultos, respectivamente. 
ERDMAN (1962) irradiou ovos com 1 a 3 horas de idade de $T$. castaneum (Herbst.) e $T$. confusum (Du Val) com raio-X. Concluiu que as doses letais foram de 1 e $2 \mathrm{krad}$, respectivamente.

DISYAN (1966) estudou os efeitos da radiação sobre os vários estádios do ciclo evolutivo de $T$. castaneum (Herbst.), verificou que a fase de ovo foi o estádio mais radiossensível. As doses causavam $100 \%$ de mortalidade de ovos, larvas e pupas e adultos foram respectivamente: 1.530, $2.200,6.200$ e $62.000 \mathrm{rad}$. A dose esterilizante para adultos foi de $6.000 \mathrm{rad}$.

BROWER \& DAVIS (1973) irradiaram ovos de $T$. castaneum (Herbst.) com uma dose de $2,5 \mathrm{krad}$, sob uma taxa de dose de 6; 18 e 43, $\mathrm{krad} / \mathrm{seg}$, em diferentes idade (18 $\pm 6,42 \pm 6 \mathrm{e}$ $66 \pm 6$ horas), observaram que os ovos com dois dias de idade ( $42 \pm 6$ horas) foram mais sensíveis à radiação gama. A eclosão das larvas quando os ovos foram irradiados com uma taxa de dose de 43 $\mathrm{rad} / \mathrm{seg}$ foi menor que os irradiados com uma taxa de dose de 6 a $18 \mathrm{rad} / \mathrm{seg}$.

MEHTA \& SETHI (1994) realizaram pesquisas para determinar a suscetibilidade de $T$. castaneum (Herbst.) à radiação gama, observaram que a radiossensibilidade de ovos diminuiu com o aumento da idade do embrião; ovos com idade de 72-96 horas necessitaram de uma dose de radição elevada para serem esterilizados.

HU et al. (1985) verificaram os efeitos da radiação gama sobre $T$. castaneum (Herbst.), e observaram que, com três semanas após a irradiação, não houve sobrevivência de pupas e adultos com doses de $200-600 \mathrm{~Gy}$, e todos que receberam uma dose de $100 \mathrm{~Gy}$ estavam mortos. A dose de 41 Gy matou $100 \%$ de ovos e larvas.

METHA et al. (1990) irradiaram ovos de T. castaneum (Herbst.) de grupos de idade diferentes, verificaram que a dose de radiação gama de 6 krad impediu a eclosão de larvas, em ovos com idades de 2-3 dias, e a dose de $8 \mathrm{krad}$ produziu resultados similares para ovos com idade de 3 a 4 dias. O tratamento com a dose de $10 \mathrm{krad}$ provocou a mortalidade de $100 \%$ dos ovos dois grupos.

\section{MATERIAL E METTODOS}

O presente trabalho foi desenvolvido na Seção de Entomologia do Centro de Energia Nuclear na Agricultura - USP, Piracicaba, SP.

Para a irradiação dos ovos foi utilizada uma fonte de Cobalto-60 $\left({ }^{60} \mathrm{Co}\right)$, tipo Gammabeam650 , sob uma taxa de dose de $1,28 \mathrm{kGy}$ hora.
Para obtenção de ovos, colocou-se insetos adultos provenientes da criação estoque, em vidros transparentes contendo como substrato germen de trigo, que foi peneirado 72 horas após a colocação dos insetos adultos, facilitando assim a coleta dos ovos que foram irradiados posteriormente.

Para este ensaio utilizou-se 7 tratamentos com 4 repetições contendo 10 ovos, com idade de 0 a 72 horas cada, que foram colocados em pequenos tubos de vidros para irradiação e posteriormente transferidos para placas de Petri, com $8 \mathrm{~cm}$ de diâmetro por $2 \mathrm{~cm}$ de altura. As doses de radiação gama utilizadas foram as seguintes: 0 (testemunha), $10,20,30,40,50$ e 60 Gy. Após a irradiação, as placas de Petri foram colocadas em câmara climática com temperatura de $25 \pm 2^{\circ} \mathrm{C}$ e umidade relativa de $70 \pm 5 \%$. Diariamente era feita a contagem das larvas eclodidas, e estas colocadas em vidrinhos de 3,5 cm de altura por $3,5 \mathrm{~cm}$ de diâmetro, com tampas plásticas perfuradas, onde aguardou-se o desenvolvimento at $\epsilon$ a fase adulta e geração filial.

\section{RESULTADOS}

$\mathrm{Na}$ TABELA 1, constam os valores médios de ovos irradiados, com doses crescentes de radiação gama, de T.castaneum (Herbst.), número de larvas eclodidas, pupas e adultos emergidos com sua respectiva geração filial (F-1). Com esses dados, construiram-se as Figuras 1 e 2, onde constam as médias de larvas, pupas, adultos e geração filial F-1 de $T$. castaneum (Herbst.) irradiados com doses crescentes de radiação gama na fase de ovo.

\section{DISCUSSÃo}

Conforme a literatura consultada pode-se observar que existem algumas variações sobre as doses letais e esterilizantes para esta fase do ciclo evolutivo do inseto em estudo. Isso provavelmente devido a metodologia utilizada, taxa de dose de irradiação, idade da fase do ciclo evolutivo do inseto e condições climáticas, que são os fatores mais importantes que contribuem para que ocorra discrepâncias nos resultados.

Considerando-se os efeitos da radiação gama (TABELA 1 e Figura 1), observou-se que a dose letal para inibir a total eclosão das larvas (DL100) foi a de $30 \mathrm{~Gy}$, enquanto que a (DL50) obtida graficamente a partir da porcentagem de larvas eclodidas foi a de 12,5 Gy (Figlira 2). A esterilização de adultos oriundos de ovos irradiados foi obtida com a dose de $20 \mathrm{~Gy}$. 
TABELA 1. Viabilidade de ovos, larvas e pupas e emergência de adultos de Tribolium castaneum irradiados na fase de ovo com radiação gama do Cobalto-60, para os tratamentos e sua respectiva geração filial (F-1). Médias de 4 repetições.

\begin{tabular}{|c|c|c|c|c|c|}
\hline $\begin{array}{c}\text { Doses } \\
\text { (Gy) }\end{array}$ & $\begin{array}{c}\mathbf{n}^{\circ} \text { Ovos } \\
\text { Irradiados }\end{array}$ & $\begin{array}{c}\mathbf{n}^{\bullet} \text { Larvas } \\
\text { Eclodidas }\end{array}$ & $\begin{array}{c}\mathbf{n}^{\bullet} \\
\text { Pupas }\end{array}$ & $\begin{array}{c}\mathbf{n}^{\bullet} \text { Adultos } \\
\text { Emergidos }\end{array}$ & $\begin{array}{c}\text { Média de } \\
\text { F-1 }\end{array}$ \\
\hline 0 & 10 & 8,00 & 6,75 & 6,00 & 870,75 \\
\hline 10 & 10 & 6,00 & 5,25 & 4,00 & 308,50 \\
\hline 20 & 10 & 1,50 & 1,25 & 0,75 & 0,00 \\
\hline $30,40,50 \mathrm{e} 60$ & 10 & 0,00 & 0,00 & 0,00 & 0,00 \\
\hline
\end{tabular}

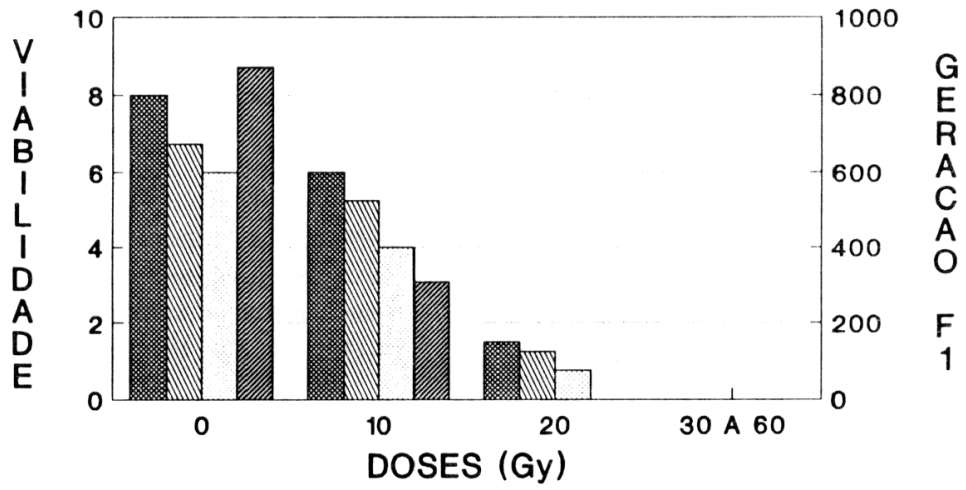

LARVAS ECLODIDAS AMIIV ADULTOS EMERGIDOS

PUPAS WIII

Figura 1. Médias do número de larvas, pupas, adultos emergidos e a geração $F_{1}$, quando ovos de Tribolium castaneum (Herbst.) foram irradiados com radiação gama do Cobalto-60.

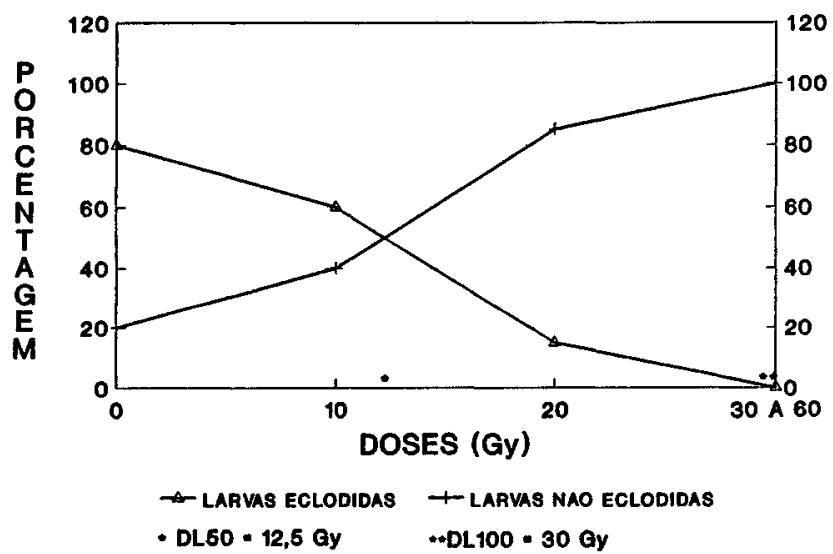

Figura 2. Porcentagem de larvas de Tribolium castaneum (Herbst.), eclodidas e não eclodidas de ovos irradiados com radiação gama do Cobalto-60. 
Os resultados obtidos para esta fase do ciclo evolutivo do inseto, estão de acordo com os de ERDMAN (1962) e (1968) e um pouco diferentes dos obtidos por MEHTA et al. (1990).

\section{CONCLUSÃO}

A DL100 e a dose esterelizante de radiação gama para os ovos de Tribolium castaneum (Herbst.) (Coleoptera:Tenebrionidae) é $30 \mathrm{~Gy}$ e $20 \mathrm{~Gy}$, respectivamente

\section{REFERÊNCIAS BIBLIOGRÁFICAS}

BROWER, J.H.; DAVIS, E.R. Sensitivity of red flour beetle eggs to gamma radition as influenced by treatment age and dose rate. Journal of Entomological Society, Tifton. v.8, n.5, p.153-157, 1973.

CROOCK, L.J. The sucetibility of the rust-red flour beetle, Tribolium castaneum (Herbst.) to gamma radiation. Berkshire, Wantage Research Laboratory, 1962. 19p. (AERE-R, 3889).

DISYAM, B. Radiation effect on redd flour beetle (Tribolium castaneum Herbst.). In: SEMINAR ON INSECT ERADICATION BY IRRADIATION, Papers, Bangkog, OAEP. p.35-41. (THAI. AEC 18).

ERDMAN, H.R. Comparative X-ray sensitivity of Tribolium confusum and $T$. castaneum (Coleoptera: Tenebrionidae) at differente developmental stages during their lifecycle. Nature. London, v.195, n.4827, p.1218, 1962.

GALLO, D. Radioisótopos no controle de pragas. $\mathbf{O}$ Solo, Piracicaba, v.1, p.30-31, 1960.
GALLO, D.; NAKANO, O.; SILVEIRA NETO, S.; CARVALHO, R.P.L.; BATISTA, G.C.; BERTI FILHO, E.; PARRA, J.R.P.; ZUCCHI, R.A.; ALVES, S.B. Manual de Entomologia Agnícola. São Paulo, 1988. 649p.

HU, T.; TSAL, L.T.; FU, Y.K. Gamma irradiation constrols Tribolium castaneum Herbst. in wheat flor. Plant Protection Bulletin, Taiwan, v.27, n.4, p.371378, 1985. Apud Review of Applied Entomology. Série: A Agricultura, 1978. (Resumo).

MEHTA, V.K.; SETHI, G.R. Effect of gamma radiation on the eggs of Tribolium castaneum (Herbst.). Journal of Nuclear Agriculture and Biology, New Delhi, v.13, n.3, p.72-74, Sept. 1984.

MEHTA, V.K.; SETHI, G.R.; GARG, A.K. Development of Tribolium castaneum (Herbst.) larvae after gamma irradiation of eggs. Journal of Nuclear Agricultural and Biology. New Delhi, v.19, n.1, p.54-57, 1990.

RUNNER, G.A. Effects of roentegen rays on the tabaco or cigarret beetle and results of new form of Röntgen tube. Journal of Agricultural Research, Tanta, v.6, n.11, p.383-388, 1916,

WIENDL, F.M. Alguns usos e efeitos das radiações gama em Zabrotes subfsciatus (Boh. 1833). Piracicaba, 1969. 205 p. Tese (Doutorado) - Escola Superior de Agricultura "Luiz de Queiroz", Universidade de São Paulo.

Recebido para publicação em 04.04.94

Aceito para publicação em 29.04.94 